\title{
Introduction to the special issue on secure communications
}

Communications security is the discipline of preventing unauthorized interceptors from accessing telecommunications in an intelligible form, while still delivering content to the intended recipients. It is often referred to by the abbreviation COMSEC. The field includes cryptosecurity, transmission security, and physical security of COMSEC equipment. COMSEC is used to protect both classified and unclassified traffic on military communications networks, including voice, video, and data. It is used for both analog and digital applications, as well as both wired and wireless links.

Following seven papers address state-of-the-art research and state-of-the-practice industry experiences are highquality articles presenting open issues, algorithms, protocols, policies, frameworks, standards, and solutions for communications related to security and privacy.

- A probabilistic model for anonymity analysis of anonymous communication networks. Jaafar Almasizadeh and Mohammad Abdollahi Azgomi.

- Using Mobile Phones to Enhance Computing Platform Trust. Wei Feng, Yu Qin, and Dengguo Feng.

- Steganalysis of BCH Code Based Stego Schemes. Natarajan V. and R. Anitha.
- ID-based Proxy Re-Signature without Pairing. Zhiwei Wang, Aidong Xia, Mingjun He.

- Age-Based Anonymity: A Randomized Routing Approach to Communication Unobservability. Peyman Taher, Alireza T. Boloorchi, M. H. Samadzadeh.

- A secure wireless mission critical networking system for unmanned aerial vehicle communications. Jahangir $\mathrm{H}$. Sarker, and Ahmed M. Nahhas.

- Remove Key Escrow from the BF and Gentry IdentityBased Encryption with Non-Interactive Key Generation. Quanyun Wei, Fang Qi, and Zhe Tang.

We express our sincere thanks to all the authors and referees for their contribution in creating this special issue. We thank the Editors-in-Chief for their encouragement and support (and friendly nudges at various stages of the process), and the administrative staff for technical help in this long process. We hope you enjoy this issue and that it inspires more research to identify and overcome future challenges in our increasingly connected world pertaining to the secure communication.

Mazdak Zamani, Azizah Abd Manaf, Isao Echizen, Shamsul Sahibuddin, Yoshiro Imai

Guest Editors 